D. F. Suen - C. K. Wang - R. F. Lin

Y. Y. Kao - F. M. Lee - C. C. Chen

\title{
Assignment of DNA markers to Nicotiana sylvestris chromosomes using monosomic alien addition lines
}

Received: 10 April 1996 / Accepted: 5 July 1996

\begin{abstract}
A sesquidiploid hybrid (PPS, $2 n=32$ ) between Nicotiana plumbaginifolia (PP, $2 n=20)$ and $N$. sylvestris (SS, $2 n=24)$ was backcrossed to $N$. plumbaginifolia to produce monosomic alien addition lines. A total of $892 n=21$ plants, each containing two sets of $N$. plumbaginifolia chromosomes and a single $N$. sylvestris chromosome, were obtained in the $\mathrm{BC}_{1}$ and $\mathrm{BC}_{2}$ generations. These plants were classified into 12 groups based on morphological characteristics. The $N$. sylvestris chromosomes in these plants were identified by RFLP and karyotype analyses. Among the 84 probes tested, 20 could not detect $N$. sylvestris-specific DNA bands, and the remaining 64 were assigned to 9 normal and 6 aberrant synteny groups. The 9 normal synteny groups corresponded to chromosomes $2,4,5$, $6,7,8,9,10$ and 12 , respectively. Four aberrant synteny groups were the result of chromosome translocations, and 2 were deletions.
\end{abstract}

Key words Nicotiana sylvestris - Restriction fragment length polymorphism (RFLP) - Chromosome addition - Synteny

\section{Introduction}

Nicotiana sylvestris belongs to section Alatae of subgenus Petunioides. It is the only species with 24 chromosomes and a symmetric karyotype in this section; all other species possess 9 or 10 pairs of chromosomes and rather asymmetric karyotypes (Goodspeed 1954). It has been hypothesized that this 12-paired species is the primitive form from which 9- and 10-paired species

Communicated by B. S. Gill

D. F. Suen - C. K. Wang - R. F. Lin - Y. Y. Kao

F. M. Lee - C. C. Chen $(\bowtie)$

Department of Botany, National Taiwan University, Taipei,

Taiwan, Republic of China arose (Goodspeed 1954). However, the mechanisms of these changes remain to be explored.

$N$. sylvestris (SS) is also the maternal parent of cultivated allotetraploid $N$. tabacum (SSTT, $2 n=48$ ) (Gray et al. 1974; Bland et al. 1985; Olmstead and Palmer 1991). Early genetic evidence indicated that in $N$. tabacum several chromosomes in the $\mathrm{S}$ genome differentiated from those of extant $N$. sylvestris (Gerstel 1963). Recently, this has been confirmed by Kenton et al. (1993) using fluorescence in situ hybridization in which $N$. tabacum chromosomes were probed with $N$. sylvestris genomic DNA. These investigators observed that the three different $N$. tabacum genotypes used in their study each had up to nine homozygous translocations between chromosomes of the $\mathrm{S}$ and $\mathrm{T}$ genomes.

Comparative genome mapping (Bonierbale et al. 1988) would be a promising approach for elucidating chromosomal structural relationships among species in the section Alatae and between N. sylvestris and $N$. tabacum. As a first step in comparative genome mapping, we have constructed $N$. plumbaginifolia-sylvestris monosomic addition lines, identified the $N$. sylvestris chromosomes in these lines and assigned DNA markers to $N$. sylvestris chromosomes. The results of these studies are presented in this paper.

\section{Materials and methods}

\section{Plant material}

Seeds of Nicotiana plumbaginifolia Viviani (PP, $2 n=20)$ and $N$. sylvestris Spegazzini \& Comes (SS, $2 n=24)$ were provided by the US Department of Agriculture, Beltsville, Md. Haploid plants of these two species were obtained from anther culture (Chen et al. 1985). Sesquidiploid hybrids PPS $(2 n=32)$ were produced from protoplast fusion (Hung et al. 1993). One sesquidiploid hybrid with no detectable chromosomal aberrations was backcrossed as female parent to $N$. plumbaginifolia, and the chromosome number of the $\mathrm{BC}_{1}$ plants was determined. Of the 516 plants analyzed, 2 had $2 n=46$ and 48 each; the others fell in the range of $2 n=20-29$, with 24 and 25 being the most frequent chromosome number and 21 
chromosomes occurring only in 7 plants. Approximately $3 \%$ of these plants also possessed a centric fragment. Six $\mathrm{BC}_{1}$ plants with $2 n=23-25$ chromosomes were selected, backcrossed again as female parent to $N$. plumbaginifolia and the chromosome number of the $\mathrm{BC}_{2}$ plants was determined. Of the 260 plants analyzed, those with 21 (96 plants) and 22 (72 plants) chromosomes were the most frequent. All $2 n=21$ plants were maintained in vitro (Huang and Chen 1988) and grown in pots in the greenhouse

\section{Chromosome preparation}

Somatic chromosomes were prepared from root-tip cells of in vitrocultured plants. Excised roots were treated with $2 \mathrm{mM}$ 8-hydroxyquinoline at $18^{\circ}-20^{\circ} \mathrm{C}$ for $2 \mathrm{~h}$ and $30 \mathrm{~min}$, fixed in ethanol-glacial acetic acid (3:1) overnight, hydrolyzed in $1 \mathrm{~N} \mathrm{HCl}$ at $60^{\circ} \mathrm{C}$ for $7 \mathrm{~min}$, stained in basic fuchsin for $1 \mathrm{~h}$ and treated with $1 \%$ pectinase for $1 \mathrm{~h}$. Root tips were squashed in $45 \%$ acetic acid.

\section{RFLP analysis}

\section{Plant DNA extraction}

Total DNA was isolated from young leaves collected from single plants of the parental species, the sesquidiploid hybrid and monosomic addition plants according to the method of Gawel and Jarret (1991) with the following modifications: (1) the extraction buffer contained $1 \%$ polyvinylpyrrolidone, (2) extracted DNA was further purified by being dissolved in $1 \mathrm{M} \mathrm{NaCl}$ and precipitated with ethanol.

\section{Source and preparation of probes}

Random genomic clones from $N$. plumbaginifolia were the main source of probes. Total plant DNA isolated from the leaf tissue was digested with the restriction enzyme Pst $\mathrm{I}$, and fragments $0.5-2.5 \mathrm{~kb}$ long were selected for ligation into the pUC18 and pUC19 plasmids. E. coli strain TG-1 was then transformed with ligated plasmids (Sambrook et al. 1989). The libraries were screened to eliminate those clones corresponding to repetitive and organellar sequences by Southern hybridization using digoxigenin-11-dUTP-(Boehringer Mannheim, Germany) -labeled N. plumbaginifolia total DNA as a probe. Six random cDNA clones from $N$. plumbaginifolia and one cDNA clone for the nitrate reductase apoenzyme (pNR) from tobacco, provided by Dr. J. C. Chen, Institute of Molecular Biology, Academia Sinica, were also used as probes. Inserts of all the clones were labeled with dig-dUTP by the polymerase chain reaction (PCR) (primers were oligonucleotides 5'-CGCCAGGGTTTTCCC AGTCACGAG-3' and 5'-TCACACAGGAAACAGCTATGAC- $3^{\prime}$; 30 cycles: $1 \mathrm{~min} 30 \mathrm{~s}$ at $94^{\circ} \mathrm{C}, 1 \mathrm{~min} 30 \mathrm{~s}$ at $55^{\circ} \mathrm{C}, 2 \mathrm{~min} 30 \mathrm{~s}$ at $72^{\circ} \mathrm{C}$; final extension at $72^{\circ} \mathrm{C}$ for $10 \mathrm{~min}$ ), in which $10 \%$ dTTP was substituted with dig-dUTP.

\section{Southern hybridization}

DNA (5-10 $\mu$ g per lane) from the parental species, the sesquidiploid hybrid and monosomic addition plants was digested with EcoRI, EcoRV, HindIII and DraI and electrophoresed on $0.8 \%$ agarose gel. Blotting, hybridization and washing were conducted as described by Kreike et al. (1990) except that SSC was replaced by SSPE. Hybridization signals were detected by chemiluminescent reaction using AMPPD (Boehringer Mannheim, Germany) as substrate according to manufacturer's instructions.

\section{Results}

Morphology of monosomic addition lines

Of the 103 monosomic addition plants obtained in the $\mathrm{BC}_{1}$ and $\mathrm{BC}_{2}$ generations, 89 survived and grew to maturity in the greenhouse. These plants closely resembled N. plumbaginifolia but could be distinguished from it as well as from each other in a number of morphological characteristics, such as growth habit, plant height, shape, size and color of leaves, shape and size of flowers (Fig. 1) and presence or absence of tillers. On the basis of these characteristics, the 89 monosomic addition plants were classified into 12 morphological groups. The number of plants in each group is shown in Table 1.

\section{Cytology of monosomic addition lines}

Haploid N. plumbaginifolia has 5 large, 3 medium and 2 small chromosomes, all of which are telocentric or acrocentric with 1 of the small chromosome possessing a satellite (Fig. 2A). The 12 chromosomes of haploid N. sylvestris are fairly uniform in length (Fig. 2B). Chromosomes 1, 2 and 12 are metacentric, but they could be distinguished by size (Fig. 2C). Chromosomes $3,4,5,7$ and 8 are submetacentric; 2 of these (chromosomes 5 and 7) have a satellite, and the rest differ from each other in arm ratio. Chromosomes 6, 9,10 and 11 are subtelocentric; chromosome 6 is characterized by having a satellite, but chromosomes 9,10 and 11 could not be distinguished from each other with certainty. The secondary constriction in chromosome 5 is less prominent compared with those in chromosomes 6 and 7 (Fig. 2C).

Identification of the $N$. sylvestris chromosomes in monosomic addition plants indicated that (1) plants of morphological group I and 1 plant in group VII contained a large metacentric chromosome (Fig. 3A, B); (2) those of groups II, X, XI and XII each had a large submetacentric chromosome (Fig. 3C-F); (3) those of groups III, IV, V, VI and 9 plants in group IX contained chromosomes $5,6,7,8$ and 12 , respectively (Fig. 3G-J, M); (4) 7 plants in group VII and all of the plants in group VIII possessed a small subtelocentric chromosome (Fig. $3 \mathrm{~K}, \mathrm{~L}$ ) and (5) 4 plants in group IX possessed a deficient chromosome designated del-1 or del-2 (Fig. 3N, O).

\section{Molecular characterization of monosomic addition lines}

Screening of 392 PstI genomic clones by Southern hybridization using $N$. plumbaginifolia total DNA as a probe revealed that $251(64 \%)$ clones contained 

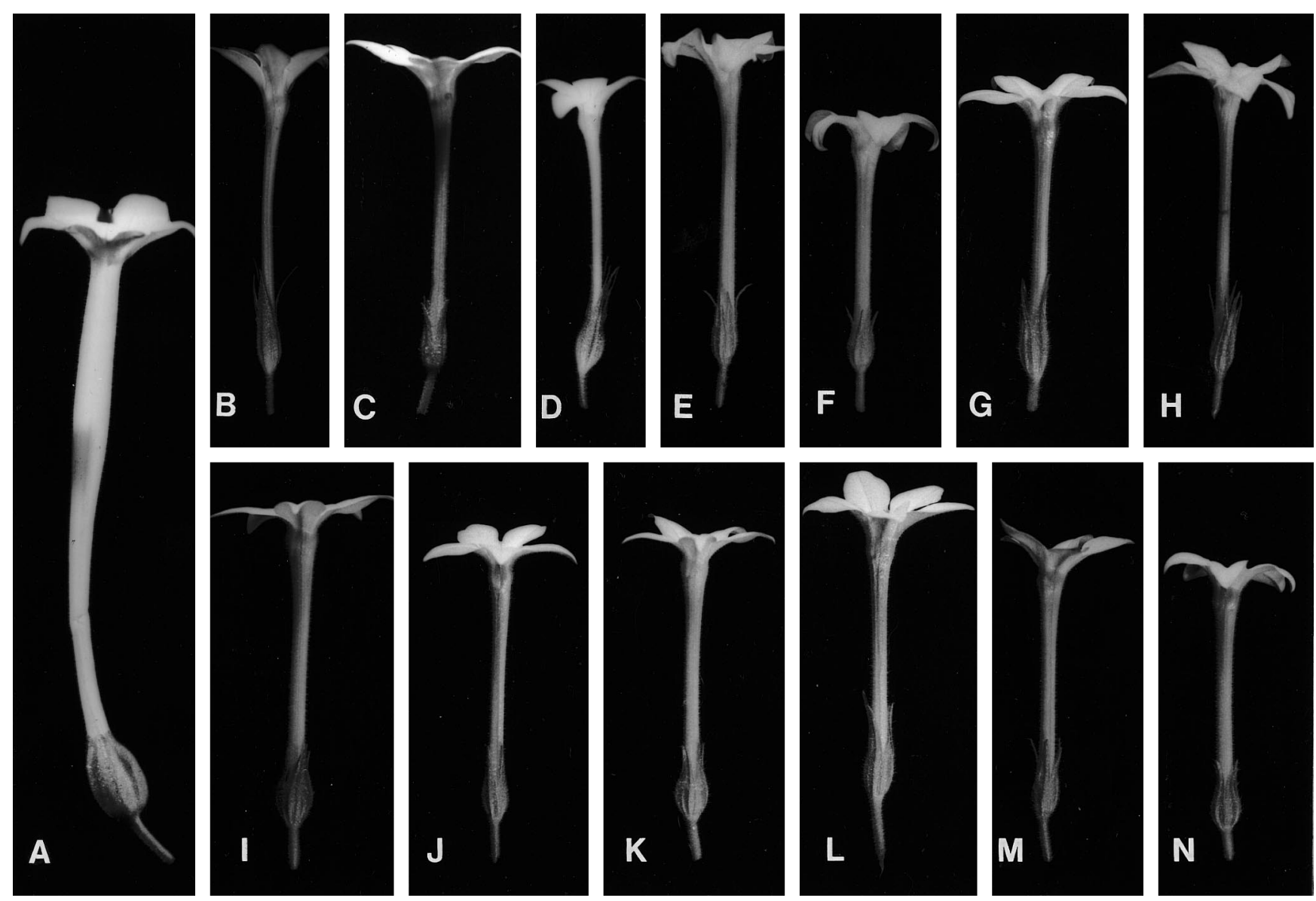

Fig. 1A-N Flowers of $N$. sylvestris (A), N. plumbaginifolia $(\mathbf{B})$, and 12 morphological groups of $N$. plumbaginifolia-sylvestris monosomic addition plants $(\mathbf{C}-\mathbf{N})$. C Plant no. 59, group I; D no. 2, group II; E no. 318-47, group III; F no. 88, group IV; $\mathbf{G}$ no. $387-4$, group V; H no. 297-25, group VI; I no. 318-2, group VII; J no. 387-26, group VIII; K no. 389-40, group IX; L no. 297-9, group X; M no. 318-24, group XI; N no. 12, group XII

repetitive or organellar sequences. The remaining 141 $(36 \%)$ clones were surveyed for copy number and polymorphism by probing them onto membranes containing DNA from $N$. plumbaginifolia and $N$. sylvestris digested with restriction enzymes EcoRI, EcoRV, HindIII and DraI. One hundred and twenty-one (86\%) clones displayed three or fewer bands and detected polymorphisms between the two Nicotiana species with at least one of the four restriction enzymes.

Figure 4 is an example of Southern blot analysis of 15 monosomic addition plants using clone 1882 as a probe. The patterns indicated that 2 plants contained at least a common $N$. sylvestris chromosome segment in which clone 1882 was located. A similar analysis was made on 89 monosomic addition plants using 77 singleor low-copy genomic clones and 7 cDNA clones as probes. Twenty genomic clones did not disclose $N$. sylvestris-specific bands in the 89 plants tested.
Results from probing with other clones are summarized in Table 1.

A total of 73 putative loci were mapped to 9 synteny groups based on 64 probes that detected N. sylvestrisspecific bands in monosomic addition plants (Table 1). Of these putative loci, 17 (23\%) were mapped to 2 or 3 synteny groups. All of the duplicated loci, except those identified by probe 18255 , were non-syntenic. Probe 18255 displayed three DNA fragments, of which 2 were syntenic and the third was in a different synteny group. The mapping of duplicated loci to 1 synteny group is attributed to the recovery of two different deletions from the same chromosome (Table 1, Fig. 5).

Monosomic addition plants nos. 297-9, 318-24 and 12 each contained DNA markers belonging to 2 synteny groups (Table 1). Plant no. 24 carried 6 markers from 1 synteny group and 1 marker of unknown origin. This plant was morphologically indistinguishable from the other plants of group VII.

\section{Discussion}

There are several reasons for choosing N. plumbaginifolia as a recipient for $N$. sylvestris chromosomes. 
Table 1 Characterization of $N$. plumbaginifolia-sylvestris monosomic addition lines

\begin{tabular}{lll}
\hline $\begin{array}{l}\text { Morphological } \\
\text { group } \\
\text { (no. of plants) }\end{array}$ & $\begin{array}{l}\text { N. sylvestris } \\
\text { chromosome } \\
\text { present }\end{array}$ & Markers assigned to the $N$. sylvestris chromosome $^{\mathrm{a}}$ \\
\hline I (3) & 2 & $1890,1893,18182,18195-1,18197,18249,1933, \mathrm{c} 161, \mathrm{c} 749-1$ \\
II (13) & 4 & $1867,1877,18150,18175,18190,1915,1919,1954-2,19119-2$ \\
III (8) & $5^{\mathrm{b}}$ & $1812,1842,18253,19119-1,19147$ \\
IV (15) & $6^{\mathrm{b}}$ & $18126,18217,1932,1943,19125,19149$ \\
V (5) & $7^{\mathrm{b}}$ & $1850,18124-2,18238,19109$ \\
VI (17) & 8 & $1874,18166-1,18173,18186-3,18203,1949,19148, \mathrm{c} 237, \mathrm{c} 525, \mathrm{pNR}$ \\
VII (8) & 9 & $1892,18124-1,18128,18166-2,18186-1,18195-2,18252,1923,1961,19136$ \\
$\quad(7)$ & Normal & $(18128,18186-1,18195-2,1923,1961,19136)^{\mathrm{c}}, \mathrm{c} 457$ \\
$\quad(1)$ & T9-? & $18110,18133,18145,18208,18241,18242,18255-2,1931,1993,19145, \mathrm{c} 378$ \\
VIII (4) & 10 & $1851,1882,18157,18162,18186-2,18243,18255-1,18255-3, \mathrm{c} 749-2$ \\
IX (13) & 12 & $1851,18255-3, \mathrm{c} 749-2$ \\
$\quad(9)$ & Normal & $1851,1882,18157,18162,18186-2,18243,18255-1$ \\
$(2)$ & Del-1 & $(1812,1842,19119-1),(1874,18166-1,18173,18203,1949,19148, \mathrm{c} 237, \mathrm{c} 525, \mathrm{pNR})$ \\
$(2)$ & Del-2 & $(18253,19147),(1851,18255-3, \mathrm{c} 749-2)$ \\
X (1) & T5-8 & $(18150,18190), 19136$ \\
XI (1) & T5-12 & \\
XII (1) & T4-9 & \\
\hline
\end{tabular}

${ }^{\text {a }}$ Order of markers is arbitrary

${ }^{\mathrm{b}} \mathrm{SAT}$-chromosome

${ }^{\mathrm{c}}$ Markers in parenthesis belong to one synteny group
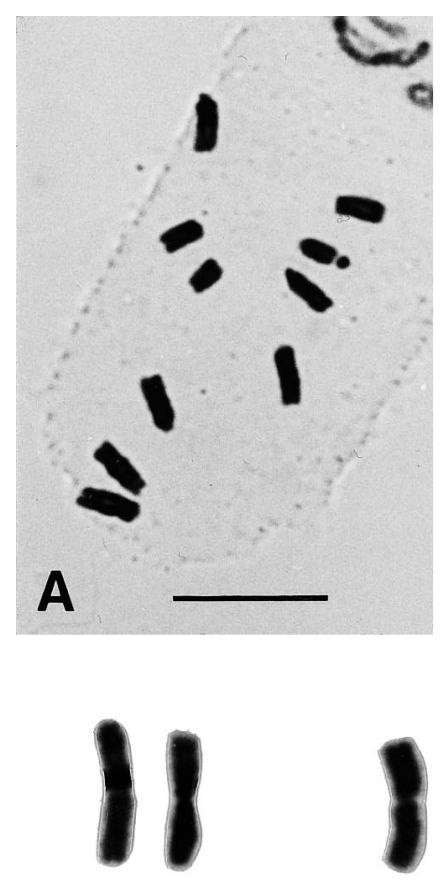

12

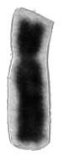

8

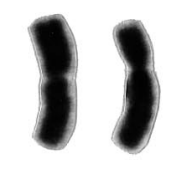

34

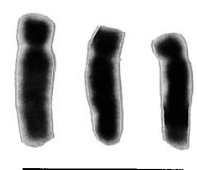

$9-11$
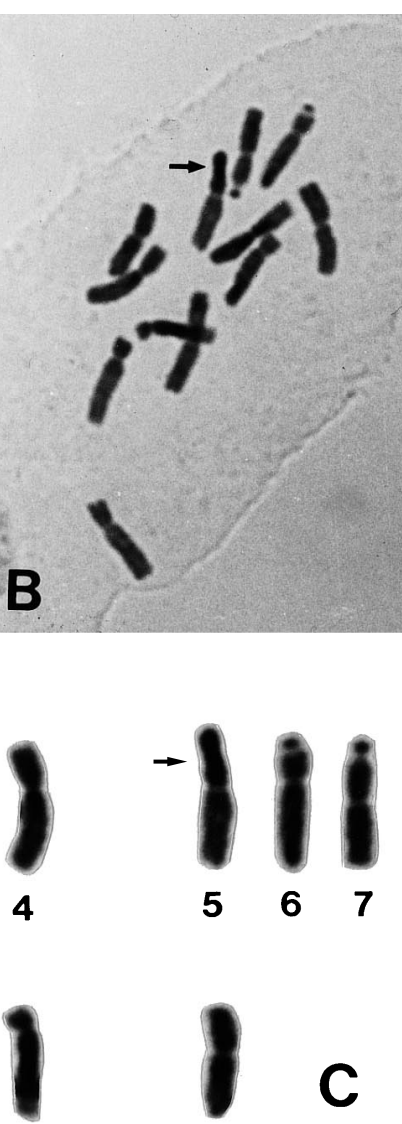

12

Fig. 2A, B Somatic metaphase chromosomes of haploid N. plumbaginifolia (A) and $N$. sylvestris $(\mathbf{B})$. Bar $=10 \mu \mathrm{m}$. C Karyotype of N. sylvestris (enlargement of B). Arrows Secondary constriction of chromosome 5

First, PPS sesquidiploid hybrids are presently available in our laboratory (Lin and Chen 1990; Hung et al. 1993) which, when crossed as female parent to N. plumbaginifolia, should give rise to monosomic addition plants. Second, the striking difference in karyotype between these two species (Goodspeed 1954; Lin and Chen 1990) makes it easy to identify the $N$. sylvestris chromosomes in hyperploids and monosomic addition plants. Third, there is little homology between the genomes of these two species (Lin and Chen 1990), therefore, the chances for intergenomic recombination would be low during the generation of monosomic addition lines.

A total of 89 N. plumbaginifolia-sylvestris monosomic addition plants were obtained in this study. Based on chromosome morphology and synteny relationships of DNA markers, we classified these plants into 9 nomal and 6 aberrant lines. The $N$. sylvestris chromosomes in the normal lines were chromosomes 2 , $4,5,6,7,8,9,10$ and 12 , respectively. The number of markers on these chromosomes ranged from 4 for chromosome 7 to 11 for chromosome 10. The absence of chromosomes 1, 3 and 11 monosomic addition lines may be explained by the fact that either these lines were inviable or the hyperploid derivatives of PPS selected

Fig. 3A-O Somatic metaphase chromosomes of N. plumbaginifoliasylvestris monosomic addition plants. A no. 59, group I; B no. 24 , group VII; C no. 2, group II; D no. 297-9, group X; E no. 318-24, group XI; F no. 12 , group XII; G no. $318-47$, group III; H no. 88 , group IV; I no. 387-4, groupV; J no. 297-25, group VI; K no. 318-2, group VII; L no. 387-26, group VIII; $\mathbf{M}$ no. 389-40, group IX; $\mathbf{N}$ no. 389-4, group IX; O no. 389-49, group IX. Arrows N. sylvestris chromosomes in monosomic addition plants. Bar, $10 \mu \mathrm{m}$. 

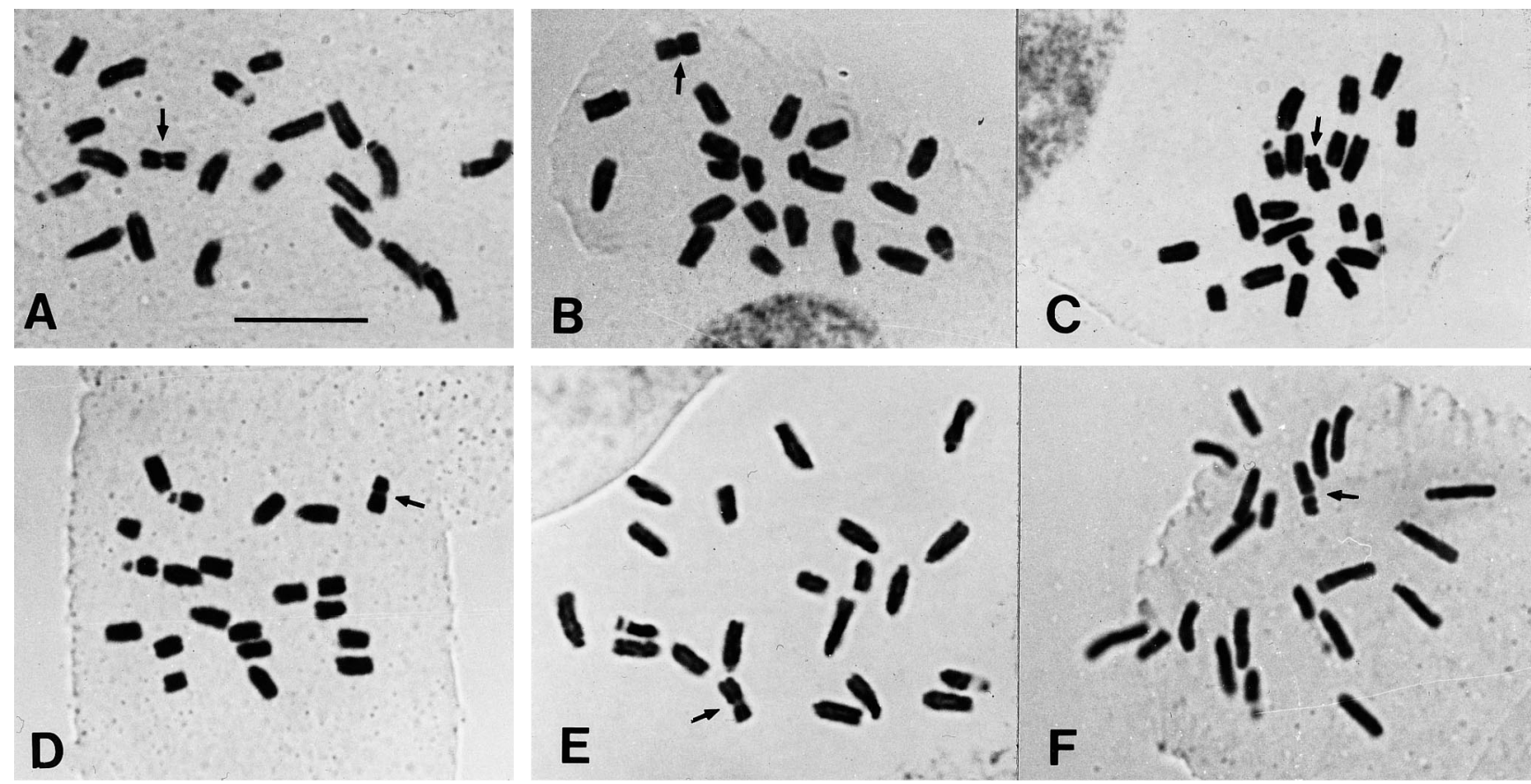

D

$-10-12$

$\because-\frac{1}{3}=$

D

$-x^{8} 8$

G
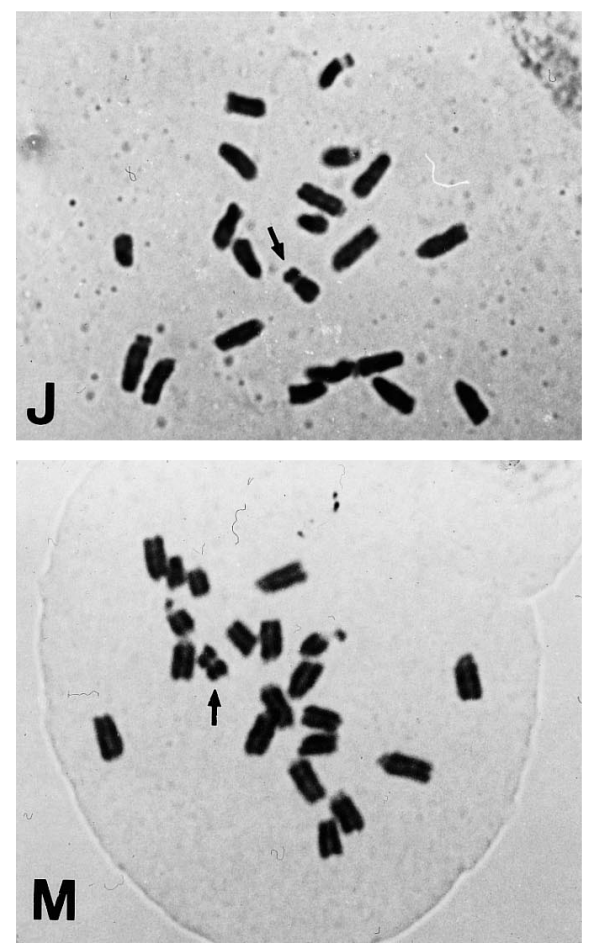

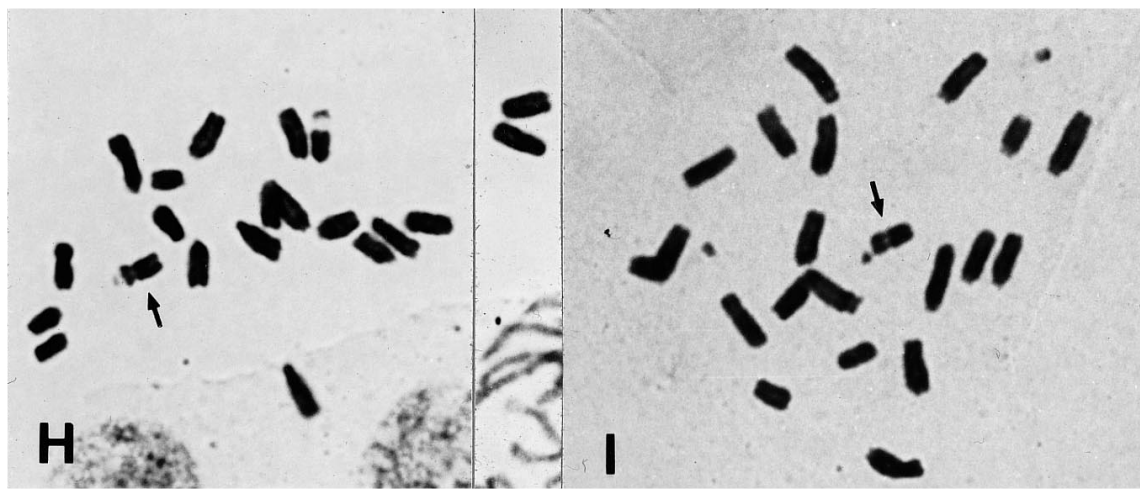

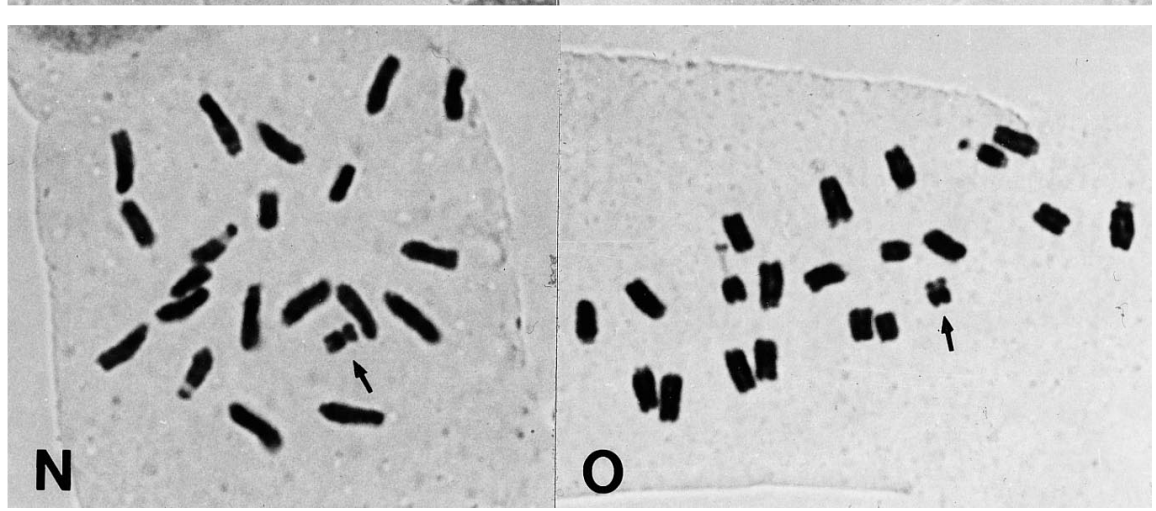


$\mathrm{kb}$

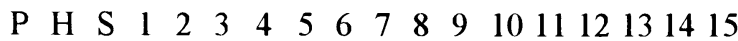

20

8.2

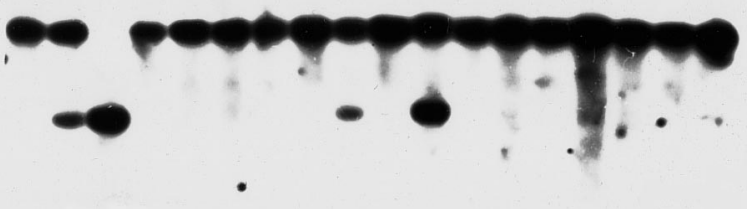

Fig. 4 Southern blot analysis of $N$. plumbaginifolia $(P)$, PPS sesquidiploid hybrid $(H), N$. sylvestris $(S)$ and monosomic addition plants $(1-15)$. Genomic DNA was digested with DraI and probed with 1882. N. sylvestris-specific fragment can be seen in lanes 6 and 8
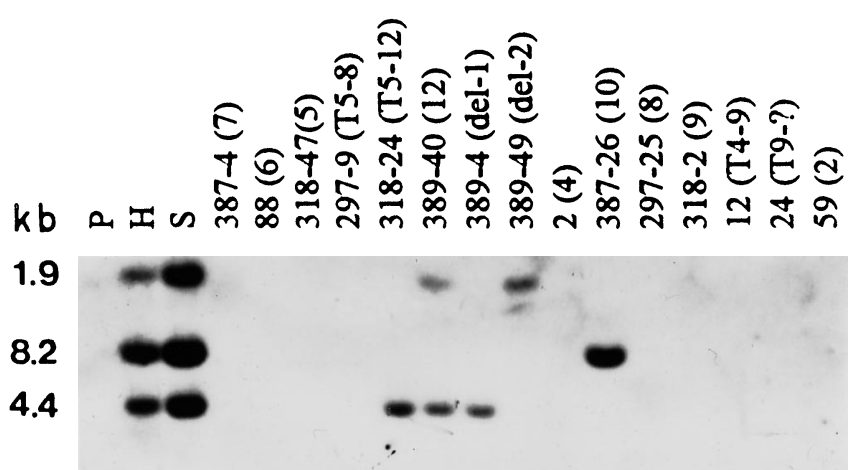

2.0

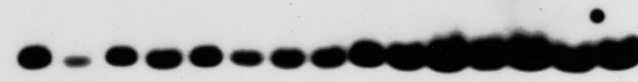

Fig. 5 Southern blot analysis of $N$. plumbaginifolia $(P)$, PPS sesquidiploid hybrid $(H), N$. sylvestris $(S)$, and monosomic addition plants (nos. 387-4, 88, 318-47, 297-9, 318-24, 389-40, 389-4, 389-49, 2 , 387-26, 297-25, 318-2, 12, 24 and 59). Genomic DNA was digested with DraI and probed with 18255 . This probe detected three fragments in the $N$. sylvestris genome, two located in chromosome 12 and one in chromosome 10 . The $N$. sylvestris chromosomes in monosomic addition plants are indicated in parenthesis

for the generation of monosomic addition lines did not contain these chromosomes.

Four monosomic addition plants carried DNA markers belonging to $2 \mathrm{~N}$. sylvestris synteny groups (Table 1). In these plants, the $20 \mathrm{~N}$. plumbaginifolia chromosomes appeared to be normal, but the added chromosomes were dissimilar to the $N$. sylvestris chromosomes that contributed DNA markers. The chances for DNA introgression from $N$. sylvestris to the $N$. plumbaginifolia genome during the generation of monosomic addition lines would be low, as no trivalent formation has been observed in microsporocytes of PPS sesquidiploid hybrids (Lin and Chen 1990). Therefore, the simplest explanation would be that each of these plants carried a translocated $N$. sylvestris chromosome. Based on the DNA markers they carried, we designated the translocated chromosomes in 3 plants T5-8, T5-12, and T4-9, respectively. The translocated chromosome in the fourth plant involved chromosome 9 and 1 chromosome not present in the normal monosomic addition lines obtained in this study.

Two aberrant lines, del-1 and del-2, carried 3 and 7 DNA markers, respectively, from $N$. sylvestris chromosome 12 (Table 1). The added chromosomes in these lines were smaller compared to the normal 12. Judged from the DNA markers they carried, we infer that each of these lines contained a terminally deficient chromosome 12. Because the deficient chromosomes were stable in somatic tissues and were transmitted sexually (data not shown), it is conceivable that their broken ends were healed by the addition of telomeric sequences (Werner et al. 1992).

Of the 12 breakpoints occurring in the $N$. sylvestris genome, 5 were in chromosome 12, 2 in chromosome 5 and 9,1 in chromosome 4 and 8 , and 1 was not identified. The distribution of DNA markers in the aberrant lines involving chromosome 12 (Table 1) suggests that the 5 breakpoints may occur only in two regions, each located in a different arm, of this chromosome. It appears that the distribution of breakpoints throughout the $N$. sylvestris genome may not be random; some regions in chromosome 12 are particularly susceptible to breakage.

The recovery of aberrant monosomic addition lines reveals the instability of $N$. sylvestris chromosomes in the genetic background of $N$. plumbaginifolia. Based on a survey of literature, Smith (1968) pointed out that chromosome instability is a common phenomenon in interspecific Nicotiana hybrids, especially when one parent belongs to section Alatae. In addition to the deletions, translocations and centric fragments observed in this study, other manifestations of chromosome instability, such as chromosome elimination, anaphase bridges, chromosome enlargement and centric fusion, have also been reported (Ar-Rushdi 1957; Moav 1961; Gerstel and Burns 1967; Collins 1967; Lin and Chen 1990). Chromosome breakage and rearrangement following interspecific hybridization, coupled with de novo centromere formation (Rhoades and Dempsey 1966; Voullaire et al. 1993), may therefore, play an important role in chromosomal evolution in the section Alatae in which all species are diploid but they differ in basic number, karyotype symmetry and DNA content (Narayan 1987).

In this study we assigned 64 DNA markers to 9 of the $12 \mathrm{~N}$. sylvestris chromosomes. Our future work will be (1) the isolation of the 3 missing monosomic addition lines so that DNA markers can be assigned to all $N$. sylvestris chromosomes, (2) the development of chromosome banding techniques for $N$. sylvestris so that the order and physical distance of DNA markers can be determined and (3) the utilization of the synteny 
relationships of DNA markers in N. sylvestris to investigate chromosome evolution in N. tabacum.

Acknowledgements We are grateful to J.C. Chen for his generous gift of cDNA clones; to W. J. Chen, H. Y. Wu and K. F. Chung for technical assistance. This work was supported by Grant No. NSC84-2311-B-002-022 from the National Science Council, Republic of China.

\section{References}

Ar-Rushdi AH (1957) The cytogenetics of variegation in a species hybrid in Nicotiana. Genetics 42:312-325

Bland MM, Matzinger DF, Leving CS II (1985) Comparison of the mitochondrial genome of Nicotiana tabacum with its progenitor species. Theor Appl Genet 69:535-541

Bonierbale M, Plaisted RL, Tanksley SD (1988) RFLP maps based on a common set of clones reveal modes of chromosomal evolution in potato and tomato. Genetics 120:1095-1103

Chen CC, Huang CR, To KY (1985) Anther cultures of four diploid Nicotiana species and chromosome numbers of regenerated plants. Bot Bull Acad Sin 26:147-153

Collins GB (1967) Long chromosomes in a Nicotiana species hybrid. Tobacco Sci $11: 173-174$

Gawel NJ, Jarret RL (1991) A modified CTAB extraction procedure for Musa and Ipomoea. Plant Mol Biol Rep 9:262-266

Gerstel DU (1963) Segregation in new allopolyploids of Nicotiana. II. Discordant ratios from individual loci in $6 x$ (N. tabacum $\times$ N. sylvestris). Genetics 48:677-689

Gerstel DU, Burns JA (1967) Phenotypic and chromosomal abnormalities associated with the introduction of heterochromatin from Nicotiana otophora into N. tabacum. Genetics 56:483-502

Goodspeed TH (1954) The genus Nicotiana. Chronica Botanica, Waltham, Mass.

Gray JC, Kung SD, Wildman SG (1974) Origin of Nicotiana tabacum L. detected by polypeptide composition of fraction I protein. Nature 252:226-227
Huang HC, Chen CC (1988) Genome multiplication in cultured protoplasts of two Nicotiana species. J Hered 79:28-32

Hung CY, Lai YK, Feng TY, Chen CC (1993) Chloroplast segregation in somatic hybrids of Nicotiana plumbaginifolia and $N$. sylvestris having different ratios of parental nuclear genomes. Plant Cell Rep 13:83-86

Kenton A, Parokonny AS, Gleba YY, Bennett MD (1993) Characterization of the Nicotiana tabacum L. genome by molecular cytogenetics. Mol Gen Genet 240:159-169

Kreike CM, de Koning JRA, Krens FA (1990) Non-radioactive detection of single-copy DNA-DNA hybrids. Plant Mol Biol Rep $8: 172-179$

Lin RF, Chen CC (1990) Cytological studies of interspecific somatic hybrids in Nicotiana. Bot Bull Acad Sin 31:179-187

Moav R (1961) Genetic instability in Nicotiana hybrids. II. Studies of the $W s(p b g)$ locus of $N$. plumbaginifolia in $N$. tabacum nuclei. Genetics 46:1069-1087

Narayan RKJ (1987) Nuclear DNA changes, genome differentiation and evolution in Nicotiana (Solanaceae). Plant Syst Evol 157: $161-180$

Olmstead R, Palmer JD (1991) Chloroplast DNA and systematics of the Solanaceae. In: Hawkes JG, Lester RN, Nee M, Estrada N (eds) Solanaceae III. Taxonomy, chemistry, evolution. Royal Botanic Gardens, Kew, London, pp 161-168

Rhoades MM, Dempsey E (1966) The effect of abnormal chromosome 10 on preferential segregation and crossing over in maize. Genetics 53:989-1020

Sambrook J, Fritsch EF, Maniatis T (1989) Molecular cloning: a laboratory manual. 2nd edn. Cold Spring Harbor Laboratory Press, Cold Spring Harbor, N.Y.

Smith HH (1968) Recent cytogenetic studies in the genus Nicotiana. Adv Genet 14:1-54

Voullaire LE, Slater HR, Petrovic V, Choo KHA (1993) A functional marker centromere with no detectable alpha-satellite, satellite III, or CENP-B protein: activation of a latent centromere? Am J Hum Genet 52:1153-1163

Werner JE, Kota RS, Gill BS, Endo TR (1992) Distribution of telomeric repeats and their role in the healing of broken chromosome ends in wheat. Genome 35:844-848 